\title{
Desplazar las fronteras más allá del dominio del Derecho y la Economía
}

\author{
Fernando Luis Castellanos Obregón ${ }^{1}$
}

\section{Resumen}

Intentando, como lo manifestara Foucault "superar el control y los límites fijados por medio de la acción de una identidad en la producción de sus respectivos discursos" (FOUCAULT, 1972)el avance de los campos del Derecho y la Economía históricamente se ha caracterizado por procesos de diferenciación e integración, los cuales abarcan determinadas áreas relativamente cercanas, ya sea por sus objetos de estudio o por las demandas de los distintos agentes sociales, que en definitiva las integran en el quehacer. Así, aparecen campos y sub-campos intermedios que rebasan parcialmente los límites de los conocimientos disciplinarios, tanto de la Economía como del Derecho, aunque no lo hacen por completo. Para los fines que se propone esta indagación, constitutiva de nuestra trayectoria y programa de investigación, es importante e ineludible, entonces, establecer los límites de los dos campos base que la contextualizan y le sirven de marco:el Derecho y la Economía; para luego sí, poder examinar, analizar, determinar y evaluar los alcances de campos y sub-campos contemporáneos, que los tienen como objeto e intentan expandir sus rígidos moldes y mover sus fronteras de conocimiento mediante su confluencia y vinculación con la sociedad.

Palabras clave: Derecho, Economía, Derecho y economía, campo, práctica, campo de práctica, arbitrario cultural, legitimidad, frontera de conocimiento, orden jurídico económico, límite de un campo disciplinario, derecho, economía y sociedad.

\section{Moving borders beyond the domain of Law and Economics}

\begin{abstract}
Attempting, as Foucault stated, "to overcome the control and limits set by the action of an identity in the production of their respective discourses" (FOUCAULT, 1972), the advance of the fields of Law and Economics has historically been characterized by processes of differentiation and integration, which encompass certain relatively close areas, either by their objects of study, or by the demands of the different social agents, which ultimately integrate them into the task. Thus, intermediate fields and sub-fields appear that partially exceed the limits of disciplinary knowledge, both of the Economy and of Law, although they do not do so completely. For the purposes proposed by this inquiry, constitutive of our trajectory and research program, it is important and inescapable, then, to establish the limits of the two base fields that contextualize and serve as a framework: Law and Economics; Then, yes, we can examine, analyze, determine and evaluate the scope of contemporary fields and sub-fields, which have them as an object and try to expand their rigid molds and move their frontiers of knowledge through their confluence and connection with society.
\end{abstract}

Keywords: Law, Economy, Law and economics, field, practice, field of practice, cultural arbitrariness, legitimacy, border of knowledge, legal-economic order, limit of a disciplinary field, law, economy and society.

\section{Contextualización introductoria}

"Las fronteras que algunos tratadistas persisten en mantener entre los campos del Derecho y la Economía, hoy por hoy, resultan ser un sinsentido, pues, en la práctica, son más fluidas, aunque, a su vez, más complejas de lo que cualquier ortodoxia supone" (Castellanos (2017) Del Derecho a Economía con Derecho: la relación Derecho - Economía revisitada, en Revista Con-texto, n. 48, pp. 11-24. DOI: https://doi.org/10.18601/01236458.n48.03)

\footnotetext{
${ }^{1}$ Doctor en Sociología Jurídica y Política, asociado al Observatorio de la Sociedad, las Organizaciones, la Gestión, la Emigración y el Desarrollo- OBSOGED.Ha realizadoinvestigaciones y estudios sobre conflictos y dinámicas migratorias contemporáneas; desarrollo en contextos regionales; problemáticas ambientales; relaciones de poder e instituciones regionales y locales; eficacia de la protección a emigrantes, inmigrantes y desplazados; orden jurídico-económico nacional frente a las demandas del nuevo orden global; y acerca de la relación Derecho-Economía.

2““Campo es una de las principales categorías de análisis que le permiten a Bourdieu construir su teoría sociológica. Los campos son espacios sociales relativamente autónomos, en los que los agentessociales(instituciones, grupos, clases sociales) luchan por la apropiación de un capital. Los agentes ocupan posiciones dominantes y subordinadas dentro del campo. Algunos quieren dominarlo y otros mantenerlo como está. Para que un campo exista como tal, debe haber capital y lucha por la apropiación de ese capital, por ejemplo: campo político, económico, jurídico" (FLACHSLAND C. ,2003: 118)
} 
Intentando como lo manifestara Foucault "superar el control y los límites disciplinarios en la producción de sus respectivos discursos" (FOUCAULT, 1972), el avance de los campos del Derecho y la Economía históricamente se ha caracterizado por procesos de diferenciación e integración, que abarcan determinadas áreas relativamente cercanas, ya sea por sus objetos de estudio, o por las demandas de los distintos agentes sociales, que en definitiva las integran en el quehacer. Así aparecen campos, y sub-campos intermedios, que rebasan parcialmente los límites de los conocimientos disciplinarios tanto de la Economía como del Derecho, aunque no lo hacen por completo. Para los fines que se propone esta indagación,constitutiva de nuestra trayectoria y programa de investigación, es importante e ineludible, entonces, establecer los límites de los dos campos base que la contextualizan y le sirven de marco: el Derecho y la Economía; para luego sí, poder examinar, analizar, determinar y evaluar los alcances y vicisitudes de campos y sub-campos contemporáneos que los tienen como objeto e intentan, expandir sus rígidos moldes y mover sus fronteras de conocimiento, mediante su confluencia y vinculación con la sociedad.

\section{Del Derecho y la Economía: la densidad ${ }^{3}$ de la doxa ${ }^{4}$}

Cuando se reflexiona sobre el Derecho surge el interrogante de si este es ideología o ciencia. No obstante lo anterior, como Bourdieu (1991) lo manifiesta de manera sencilla,

"Decir que el Derecho es una ideología es perder de vista la lógica y el efecto específicos del Derecho. Es operar una ruptura con la representación ingenua que pretende que el Derecho es universal, como ciencia o como norma $\{\ldots\} \mathrm{La}$ pretensión de universalidad de los juristas está fundamentada, pero de un modo distinto a como la conciben ellos; no está fundamentada en una norma fundamental. Se puede rechazar el fundamento de tipo kelsesiano del Derecho como sistema normativo; una especie de proeza de la absolutización, sin quedarse en el vacío. Hay que abandonar la cuestión del fundamento, y aceptar que el Derecho, al igual que la Ciencia, el Arte [o la Economía] puede estar fundamentado únicamente en la historia, en la sociedad, sin que por ello queden aniquiladas sus pretensiones de universalidad"5(BOURDIEU, 1991: 3)

Se ha convenido en que el Derecho, como sistema de normas, históricamente ha sido el resultado de un arduo y prolongado esfuerzo de organización, coherencia y racionalidad sistemática y acumulativa en ese campo, al cual no se entra si no se posee una competencia o capacidad específicay pertinente y una cultura jurídica ${ }^{6}$.

Respecto a las condiciones necesarias para ingresar al campo del Derecho, Brigido (2002) dice de manera más específica, que son

"El dominio de la retórica jurídica y de los conocimientos jurídicos especializados, junto al acceso y cabal aprovechamiento del acervo de recursos jurídicos acumulados por las distintas generaciones; y el modo de pensar y de actuar consagrados por el canon jurídico.

\footnotetext{
${ }^{3}$ Por densidad se suele entender la cualidad de lo que es denso. En este caso, se hace referencia a la densidad de los escritos de Economía y Derecho, la cual hace que sean poco comprendidos fuera de los círculos especializados.

${ }^{4}$ En esta indagación se entiende la doxa como el conjunto de opiniones comúnmente aceptadas en un campo, es decir, como las creencias establecidas e ideas recibidas que se aceptan sin discusión.

${ }^{5}$ Traducción del autor. El texto original de Bourdieu en francés es el siguiente: "Dire que la loi est une idéologie, c'est perdre de vue la logique et l'effet spécifiques de la loi. C'est opérer une rupture avec la représentation naïve, qui prétend que la loi est universelle, en tant que science ou en règle générale [...] La prétention à l'universalité des juristes est fondée, mais d'une manière différente sur la façon dont ils la conçoivent; il n'est pas basé sur une norme fondamentale. Nous pouvons rejeter le fondement de la loi de type kelsésien comme un système normatif, une sorte d'exploit d'absolutisation, sans rester dans le vide. Nous devons abandonner la question de la fondation et accepter que la loi, comme la science, l'art (ou l'économie) puisse être basée uniquement sur l'histoire, dans la société, sans pour autant détruire ses prétentions d'universalité" (BOURDIEU, $1991: 3$ )
}

${ }^{6}$ Existe una clara y creciente conciencia entre los juristas, de la necesidad de una capacidad argumentativa convincente, desde la cual se manejen con criterioy eficacia los lazos entre la realidad sobre la que se proyecta la acción normativa y el propio orden jurídico que persigue recomponerla. Además, entre otras habilidades se le exige al jurista la capacidad de comunicar información y divulgar el conocimiento jurídico; y el empleo de un lenguaje preciso y especializado capaz de transmitir con eficacia, a quien ocupa sus servicios, los conocimientos jurídicos teóricos y prácticos. (IBÁÑEZ, 2011: 14 y s.) 
El proceso de formación jurídica que ha tenido y tiene lugar en las Universidades consagradas en el campo, juega un papel fundamental en ello, pues es en la Universidad donde se adquieren, en parte, las competencias necesarias para la práctica profesional del Derecho" (BRIGIDO.2002:120)

Ahora bien, la autonomía del campo jurídico se afirma fundamentalmente respecto de la economía. Según esto, el Derecho se configuraría como un sistema racional esto es, siguiendo a Weber

"como un sistema diferenciado e independiente de las necesidades económicas y sociales que determinan su existencia, y al mismo tiempo formal, en tanto que creado y aplicado al margen de imperativos éticos, reglas utilitarias o de conveniencia, y postulados políticos" (WEBER citado por CALVO, 2005:7).

Sin embargo, no basta decir que el Derecho se produce y se ejerce en un espacio social relativamente autónomo, en el cual los efectos de las coerciones económicas no se ejercen. Hay que recordar con Bourdieu (2000) que el campo del Derecho es no solamente una práctica social, sino a su vez

"un campo de luchas, como cualquier otro campo, por transformar las reglas del Derecho; luchas éstas que son inseparablemente luchas jurídicas y luchas económicas" (BOURDIEU, 2000: 155 y ss.)

Entonces, si bien la pretendida autonomía del Derecho se manifiesta en la intención de estar a distancia de la Economía, de separar el universo jurídico del universo de los negocios;estos dos universos confluyen no solo en el seno de la sociedad, sino también al interior del Derecho mismobajo las diversas formas que ha adoptado y adopta hoy, por ejemplo, el Derecho regulativo, al cual se refiere Calvo (2005).

Respecto al campo de la Economía, grosso modo se sigue en su referencia a Paramio (2000) quien dice:

"La Economía ha pasado a ser la reina de las Ciencias Sociales, tanto por su prestigio como por su exitoso desembarco en los campos de la Sociología, la Ciencia Política y el Derecho, debido a la sencillez y elegancia de los modelos del análisis económico frente a otras aproximaciones heterodoxas menos claras o más idiosincráticas que aparecían como alternativas al marco de la decisión racional'(PARAMIO, 2000)

Y es que hasta hace un tiempo, la Economía se definió por su objeto: la producción y el intercambio de bienes materiales. Un primer intento de ampliación de sus alcances, consistió en incluir dentro de ese objeto todos los asuntos referidos a la asignación de recursos escasos; pero entonces se incluyó casi todo, y la Economía se colocó en la posición de una ciencia total, a riesgo de perder su credibilidad.

Fue entonces, a partir de esa situación, que algunos economistas notables (Gary Becker, sobresaliendo entre ellos) expusieron que su ciencia no se caracterizaba por su objeto, sino por su método de análisis; el cual, según su opinión, podía legítimamente ser aplicado por las demás ciencias y, en particular, por las Ciencias Sociales, a todos los aspectos de la vida humana.

Dicha tesis fue operada empíricamente de manera amplía y sistematizada, particularmente por Gary Becker, cuyos trabajos fueron consagrados a nivel mundial con el otorgamiento del premio Nobel de Economía en 1992.Según Becker (1976) dice:

"The combined assumptions of maximizing behavior, market equilibrium and stable preferences, used reflentlessly and unflichingly, form the heart of the economic approach [...] They are responsible for the many theorems associated with this approach [...] Indeed, economic analysis is based on three axioms, from which several theorems of human behavior can be deduced: the principle of maximization; the self-regulation of the market and the stability of human inclinations" (BECKER, 1976: 5)

\footnotetext{
${ }^{7}$ Traducido el texto de Becker dice lo siguiente: "los supuestos combinados de maximizar el comportamiento, el equilibrio del mercado y las preferencias estables, utilizados de manera implacable y sin límites, forman el corazón del enfoque económico [...] Son responsables de los muchos teoremas asociados con este enfoque. Efectivamente, el análisis económico se basa en tres axiomas, de los cuales pueden deducirse varios teoremas del comportamiento humano: el principio de maximización; la autorregulación del mercado y la estabilidad de las inclinaciones humanas". (Traducción realizada por el autor de esta indagación, a partir de BECKER, G. (1976) TheEconomicApproachto Human Behavior, Universityof Chicago Press, Chicago, p 5).
} 


\section{Para Gary Becker}

"el enfoque económico no supone que las unidades de decisión sean necesariamente conscientes de sus esfuerzos de maximización o que puedan formular o, en su defecto, describir de alguna manera informativa las razones por las cuales su comportamiento obedece a modelos sistemáticos"8 (BECKER, 1976: 7)

\section{Y continúa Becker manifestando que}

"All human behavior can be viewed as involving participants who maximize their utility, from a stable set of preferences and accumulate and optimal amount of information and other inputs in a variety of markets. If this argument is correct, the economic approach provided a unified framework for understanding behavior that has long been sought by and eluded Bentham, Comte, Marx and others"9(BECKER, 1976: 14)

La importancia de Gary Becker, radica en que nos permite ver el paradigma del mercado como método de descubrimiento de un sistema de reglas que se supone rige todos los comportamientos humanos.

Becker pasa así, por el tamiz del Mercado, todos los aspectos de la vida humana, incluido el Derecho.

Ahora bien, como Jiménez (1997) lo manifiesta de manera precisa

"Junto al capital económico, fuente indudable del poder político y de la hegemonía social, que se reproduce hereditariamente cristalizando el poderde las oligarquías, a la par se consolida en la sociedad contemporánea un importante capital cultural ${ }^{10}$, expresado en títulos y reconocimiento, quetambién, en gran medida, se ha ido formando a través de la herencia familiar, y el cual va estableciendo una capacidad de decisión y de poder crecientes en cada vez mayor número de instancias" (JIMÉNEZ, 1997: 210)

\section{Vicisitudes de la relación entre el Derecho y la Economía: la opacidad ${ }^{11}$ de la heterodoxia ${ }^{12}$}

Dorfman (1963, 38-39) señala como de tiempo atrás, y más en el campo de la Economía que en el del Derecho, sin que en este hayan faltado (v. gr. Alf Ross, Aarnio, Garzón Valdés, Añón, García Máynez, y en el Derecho colombiano Jaime Giraldo Ángel, Andrés Botero Bernal, Germán Silva García, Cesar Rodríguez Garavito, Mauricio García Villegas o Julieta Lemaitre Ripoll, entre otros)intelectuales inquietos, que con su particular forma de enfocar sus estudios se han mostrado insatisfechos con las limitaciones y rigideces de sus respectivos campos, por lo que han

\footnotetext{
${ }^{8}$ Traducción realizada por el autor a partir de texto original de Gary Becker, que dice lo siguiente: "the economic approach does not assume that decisions units are necessarily conscious of their efforts to maximize or can verbalize or otherwise describe in any informative way reasons for the systematic patterns in their behavior". Véase BECKER, G. (1976) The Economic Approach to Human Behavior, University of Chicago Press, Chicago, p 7.

${ }^{9}$ Traducido por el autor de esta indagación, el texto de Becker dice lo siguiente: "todo comportamiento humano puede considerarse, en tanto que implica agentes que maximizan sus utilidades a partir de un conjunto estable de preferencias y que acumulan una suma óptima de información y otros inputs en una diversidad de mercados.Si esta proposición es correcta, el enfoque económico ha suministrado un marco unificado de comprensión del comportamiento, que Bentham, Comte, Marx y otros han buscado durante mucho tiempo y que no pudieron alcanzar". Véase BECKER, G. (1976) The Economic Approach to Human Behavior, University of Chicago Press, Chicago, p 14

${ }^{10}$ Tellezacota que Bourdieu introdujo en el mapa categorial de la sociedad contemporánea el concepto de capital cultural, entendido como los bienes, los conocimientos, las capacidades y/o habilidades que se adquieren a través de la familia y las instituciones escolares, incorporado al análisis social para denotar la forma específica de los bienes, sistemas y códigos simbólicos que adopta la cultura de una sociedad. Su acumulación implica una interiorización realizada por medio del trabajo pedagógico de inculcación y asimilación, como en el caso de los títulos escolares o los concursos, que tienen una autonomía relativa con relación a quien los porta. Este capital resulta de la alquimia o "magia social" que se deriva de los ritos de institución, o sea, del poder de instituir, consagrar, clasificar del que disponen las instituciones para legitimar los límites, las diferencias y jerarquizaciones que están en la base de toda organización social, como por ejemplo, para nuestro caso, la diferencia entre el profesional y el lego.Véase TÉLLEZ IREGUI, G. (2002) Pierre Bourdieu Conceptos básicos y construcción socioeducativa. Claves para su lectura, Universidad Pedagógica Nacional, Bogotá.

${ }^{11}$ El concepto de Opacidad aquí se entiende como falta de claridad o transparencia.

${ }^{12}$ Aquí se entiende el concepto de heterodoxia como el desacuerdo o disconformidad con los principios de una doctrina o con las normas o prácticas tradicionales, aceptadas por la mayoría como las más adecuadas en un determinado campo.
} 
querido "ampliar sus límites tanto en extensión como en profundidad" (ibídem), para lo que se mostraron especialmente interesados tanto "en el reconocimiento de la relación entre las instituciones legales y la economía"(Ibídem) como

"en el énfasis en una investigación empírica más rigurosa, especialmente mediante el uso de documentos históricos, la recolección sistemática de datos estadísticos y el desarrollo de técnicas estadísticas" apoyados en la Antropología y la Sociología"(DORFMAN 1963, 38-39).Las principales vicisitudes de la relación entre el Derecho y la Economía pasan, entonces, no solo por la opacidad de la heterodoxia, sino también por las dificultades manifiestas en la aceptación de la legitimidad de dicha relación y la preocupación por la cientificidad del conocimiento jurídico.

\section{- La opacidad de la heterodoxia}

Como conceptualmente se ha precisado, se hace referencia aquí a la falta de claridad y de transparencia respecto de la relación entre el Derecho y la Economía, es decir, a las manifestaciones de desacuerdo y disconformidad con dicha relación. Cabe recordar con García (2010) que

"con el renacimiento de las ideologías conservadoras y el consecuente desmantelamiento de la política social, es entonces cuando la apertura del Derecho hacia las Ciencias Sociales dirige su mirada a la Economía y da pie al movimiento Derecho y Economía, percibido en ese entonces, como La única ruta segura para la promoción y ejercicio del Derecho"(GARCÍA 2010:43)

Fue entonces que, como contracorriente, la idea de crear un derecho más adaptable a los problemas sociales, tímidamente fue tomando fuerza.

\section{-Del arbitrario cultural desprovisto de aceptación a la legitimidad}

De otra parte, la discusión sobre la relación entre el Derecho y la Economía tienerelación con hechos y procesos socioculturales

"cuya existencia, de hecho, más no de derecho, justifica su aceptación o imposición; es decir, que están desprovistos de legitimidad, o sea, de ese arbitrio que da vigencia, reconocimiento, aceptación o que justifica toda dominación (ya sea en nombre de Dios, la naturaleza, la razón, el derecho, etc.). El conjunto de significaciones (códigos y sistemas simbólicos) que definen objetivamente la cultura de una sociedad, es arbitrario en la medida en que una estructura y su función no pueden deducirse de ningún principio universal: físico, biológico o espiritual; es decir, no dependen ni de la naturaleza de las cosas, ni de la naturaleza humana. El arbitrario cultural dominante en una sociedad corresponde, en términos generales, al de los grupos o clases sociales en posición de dominio" (TÉLLEZ, 2002: 201)

\section{- La realidad contemporánea: preocupación por la cientificidad del conocimiento jurídico.}

Tal como Montoro Ballesteros (1999) nos lo recuerda

"Ha sido casi una constante de la historia del pensamiento jurídico la duda, cuando no la negación, de la "cientificidad" de la jurisprudencia (conocimiento jurídico) y el consiguiente complejo de inferioridad de los juristas frente a los cultivadores de las llamadas ciencias positivas $\{. .$.$] De ahí que, a partir sobre todo de mediados del siglo XIX, se$ replantee, de forma persistente, la idea de aplicar al estudio del Derecho sus métodos, con el fin de elevar dichos estudios a la condición de "verdadera ciencia". La realización de dicha tarea exigió entre otras formalidades: la depuración del Derecho de todo contenido de carácter metafísico;igualmente, su reducción a la condición de mero hecho empírico; y coherentemente con ello, la tarea de comprender y explicar el Derecho en función de los principios y categorías conceptuales propios de las ciencias positivas (Lógica, Física, Sociología,Economía) [...] para configurarse como una mera regla técnica de organización social caracterizada por su neutralidad axiológica; la cual convierte al Derecho, entendido como regla técnica, en un objeto susceptible de ser tratado y estudiado con los métodos propios de las ciencias positivas.[...] Desde estos supuestos, hay que entender y valorar, por ejemplo, la significación y el alcance del empeño científico, interdisciplinar, representado por esa moderna corriente del pensamiento norteamericano conocida bajo la denominación de Análisis Económico del Derecho. [...] Las raíces de este movimiento las encontramos en los trabajos de F. Knight, H. Simon, M. Friedman, G Becker, J. Buchanan, R Coase, G. Calabresi y R Posner" (MONTORO BALLESTEROS, 1999: 425-427)

\section{La dinámica de los campos Del Derecho y la Economía: lo que hay que explicitar ${ }^{13}$}

\footnotetext{
${ }^{13}$ El concepto Explicitar aquí se entiende cómo hacer explícito, es decir, exponer con claridad.
} 
Un aspecto fundamental que vale la pena explicitar, es la vinculación entre el campo del Derecho y el campo del Poder (político, social, económico). Se puede decir con Ana María Brigido (2002) que

"El campo jurídico ocupa una posición crucial dentro del campo del Poder. Es este quien mediante el peso relativo concedido al "gobierno de la Ley" asigna y legítima los límites estructurales a la eficacia de la acción y de las normas jurídicas. El Derecho consagra el orden establecido al consagrar una visión de este orden, que es una visión de Estado, garantizada por el Estado.Así, el discurso jurídico tiene el poder de producir efectos prácticos de vital importancia para la vida de cada ciudadano y la convivencia social en general" ${ }^{, 14}$ (BRIGIDO, 2002:121).

Ahora, en cuanto a la Economía, y pese a las frecuentes críticas acerca de la falta de verosimilitud de sus análisis, suele señalarse que por lo general "toda acción económica es acción social, por lo cual toda la economía es siempre también realización de la sociedad"15. Se diría con Paramio (2002) que entonces la Economía se ha convertido

"En el principio de realidad de nuestras sociedades, y los economistas en los interpretes de esta realidad" (PARAMIO, 2002)

Al parecer, aunque este hecho se asocie con la primacía de la Economía dentro de las Ciencias Sociales,

"No se explica solo por ella, sino también por la generalización de la visión del mundo en términos de costo-beneficio" (PARAMIO, 2002)

En ese contexto, destaca el escaso espacio que en el campo de la Economía se otorga a la crítica, en especial a una que tome como base de su criterio, valores. De lo que se trata, según Judt (2010) es de que

"Durante años hemos hecho una virtud de la búsqueda del beneficio material: de hecho, esta búsqueda es todo lo que queda de nuestro sentido de un propósito colectivo. Sabemos qué cuestan las cosas, pero no tenemos idea de lo que valen. Ya no nos preguntamos sobre un acto legislativo o un pronunciamiento judicial ¿Es legítimo? ¿Es ecuánime? ¿Es justo? ¿Es correcto? ¿Va a contribuir a mejorar la sociedad o el mundo en el cual vivimos?” (JUDT, 2010)

\section{La razón de la crítica tiene que ver aquí con el hecho de que, por una parte,}

"toda cultura es arbitraria (se constituye en arbitrariedad cultural), es decir: es producto del trabajo de los hombres. No hay manifestaciones culturales que emanen de la naturaleza. Lo que sí existen son manifestaciones culturales arbitrarias provenientes de determinadas sociedades o de determinadas clases sociales, que se imponen como naturales, evidentes y objetivas. Esta operación es la que realiza la institución escolar al imponer la cultura de una sociedad o de una clase social particular como la cultura universalmente válida" (FLACHSLAND, C., 2005: 218)

Y más aún, como Téllez (2002) bien lo manifiesta, cuando

“el proceso de legitimación que instaura la legitimidad, es complejo" (TÉLLEZ, 2002: 211)

Y además de ser complejo, emplea las más variadas estrategias que lo que pretenden, en última instancia, como lo manifiesta Téllez (2002) es

"el mantenimiento del "orden establecido" haciendo desaparecer (ocultando) el carácter arbitrario de la dominación" (TÉLLEZ, 2002:211)

Este proceso, como Téllez (2002) lo manifiesta, termina convirtiendo

"las relaciones de fuerza en relaciones de sentido; conversión esta que es, justamente, lo que otorga una legitimidad" (TÉLLEZ, 2002: 211)

Entonces, quiere esto decir que la razón del poder ya no se busca más en una instancia soberana que trasciende la sociedad, sino en reglas de funcionamiento inherentes a esta. Y a partir de allí, como Supiot (2007) lo acota

\footnotetext{
${ }^{14}$ El campo del poder, se entiende aquí en el sentido asignado por Bourdieu como un campo de fuerzas, el cual está definido por la estructura de la correlación de fuerzas existente entre formas de poder o entre diferentes especies de capital. Es también, de manera simultánea, un campo de luchas por el poder entre distintos detentadores de diferentes formas de poder. Detentar se refiere a retener algo contra derecho. Véase BRIGIDO, Ana María "Bourdieu y sus aportes a una sociología del campo jurídico" en Revista Páginas N²-3(2) U. N. C. Argentina, pp 119-122, Septiembre de 2002
}

${ }^{15}$ LUHMANN N (2017) La economía de la sociedad, Herder, Ciudad de México 
"la cuestión del poder ya no se plantea en términos de gobierno soberano, sino de gobierno eficaz" (SUPIOT, 2007:212)

\section{- Pensar los límites ${ }^{16}$}

Ahora bien, en cuanto hace referencia a sus límites, el campo del Derecho no llegó nunca a ser considerado del todo una ciencia social. Ante todo, ha estado siempre estrechamente vinculado a su función tradicional de preparar abogados.

Por ello, los científicos sociales por lo general vieron la jurisprudencia con escepticismo. Les parecía un campo demasiado normativo y con demasiada poca raíz en la indagación empírica. Ante la exigencia de un concepto de Derecho, acota Javier de Lucas en nota introductoria al libro Funciones del Derecho de Ferrari (2014) que este autor italiano propone

"una "razonable" explicación de lo que es el derecho, pese al reconocimiento del carácter "indefinible" de sus límites, cuando subraya algo ya advertido por Durkheim ${ }^{17}$ y en lo que insiste Habermas ${ }^{18}$ : su dimensión simbólica y comunicativa, el carácter de mensaje que tienen las normas jurídicas (en su sentido amplio, kelsesiano: también las sentencias judiciales y los actos administrativos). El Derecho es, a la vez escenario de confrontación de las acciones de los sujetos (esto es, de sus proyectos) e instrumento de tales proyectos (y no solo en el clásico, formal-legalista, sentido descendentelascendente, característico de la teoría del Derecho del positivismo formalista), "regla persuasiva que trata de orientar expectativas y conductas". En definitiva, un sistema abierto, ya que los proyectos en cuestión son cambiantes y el Derecho depende de las operaciones semánticas y pragmáticas de los sujetos" (FERRARI, 2014:21) ${ }^{19}$

Sin embargo, con mirada antropológica Alain Supiot (2007) observa el Derecho y la evolución de las leyes

"Como un recurso por el cual, a través del sometimiento colectivo a un orden existente, los hombres construyen un sentido (compartido, además) para su propia vida" (SUPIOT, 2007: 300)

\section{- Repensar los límites: crítica de la razón establecida}

Al respecto, Carlos Lista y Ana María Brigido (2002) acotan como

"Lo jurídicamente "pensable" incluye aseveraciones tales como la que sostiene que el Derecho es un sistema normativo que refleja y genera orden; la que reduce el fenómeno jurídico al Derecho y éste a la legalidad vigente; la referente a aceptar sólo la crítica jurídica orientada a mejorar el sistema jurídico; la que afirma que el orden y los textos jurídicos son autónomos y autosuficientes y la regla fundamental que prescribe que el Derecho tiene un carácter formal.Lo "impensable" constituye, entonces, aquello que no es considerado propio de esta esfera de acción, y por lo tanto, poco legítimo como contenido de enseñanza, en lo que se incluyen ideas tales comoque el derecho es un sistema normativo próximo o dependiente de prácticas sociales, usos y costumbres; o que es una construcción social e histórica, consecuencia de las estructuras de poder y de la influencia de acontecimientos y procesos sociales, políticos y económicos. También resulta impensable justificar o evaluar al Derecho de manera explícita por referencia a valores e intereses o por las consecuencias políticas, económicas o sociales que tiene la ley y su aplicación" ${ }^{\text {20 }}$ (LISTA C. Y BRIGIDO A. M., 2002: 287)

\footnotetext{
${ }^{16}$ Aquí se toma el concepto de límite como la divisiónsimbólica entre disciplinas que tienen alguna relación entre sí. Su fin o alcance.

${ }^{17} \mathrm{Cfr}$., por ejemplo, en el clásico libroDe la división du travail social, PUF, París

${ }^{18}$ Cfr., por ejemplo, en su libro Teoría de la acción comunicativa, traducción al castellano M. Jíménez, Madrid, Taurus, 2 volúmenes, 1987-1988; en su artículo "Legitimidad a través de la legalidad”, traducción al castellano M. Jíménez, aparecida en Doxa, vol V, pp. 21 a 47; o en su otro libro Facticidad y validez: sobre el derecho y el estado democrático de derecho en términos de teoría del discurso, editorial Trotta, 2010, Madrid.

${ }^{19}$ VéaseJavier de Lucas nota introductoria a la edición castellana de FERRARI, V. Funciones del Derecho, Bogotá, Universidad Externado de Colombia

${ }^{20}$ Véase CARLOS LISTA Y ANA MARÍA BRIGIDOLa enseñanza del Derecho y la formación de la conciencia jurídica, Sima Editora, Córdoba, octubre de 2002.
} 


\section{La necesidad de la interdisciplinariedad ${ }^{21}$ o ¿transdisciplinariedad ${ }^{22}$ ?}

"No sé por qué razón, el ser humano tiene una rara tendencia a negar la existencia de aquello que difícilmente domina. Y lo hace renunciando al conocimiento, o destruyendo el ser que no se deja o no es capaz de dominar. Debe ser eso lo que está ocurriendo con la política: resulta más fácil reemplazarla por un sistema jurídico, que aparentemente es autosuficiente, completo, hermético, y que no la necesita para nada. Que contiene la verdad revelada. Que es el punto de llegada. Que no admite contradictorios. Pero ese sí que es un inmenso error. Esa sí que es una gran equivocación. Porque cuando se cede el espacio de lo político para sustituirlo por lo normativo, como si aquél no hubiera existido nunca o hubiera dejado de existir, no se repara en que lo que se está haciendo es trasladar (con el pretexto de la naturaleza del sistema jurídico) a otro sujeto el ejercicio de lo político. Así es como en las sociedades, lenta e imperceptiblemente, se está ignorando el poder de decisión de las comunidades, concentrándolo en cabeza de grupos de expertos que nos llamamos juristas, utilizamos un lenguaje de juristas y para juristas, y tenemos procedimientos que solo conocemos los juristas. ¡Como si los demás no contaran!! (MEJIA ESCOBAR, C. Prólogo a la segunda edición de GIRALDO ANGEL JAIME et aliaMetodología y técnica de la Investigación sociojurídica)

En referencia al asunto, García y Rodríguez (2003) resaltan que

"La interdisciplinariedad en el análisis del Derecho hace alusión al cuestionamiento de las fronteras artificiales entre los distintos campos de conocimiento impuestas por la tradición de la ciencia moderna. De lo que se trata es de subvertir el orden establecido por la especialización científica propia de la modernidad [...] cuyos practicantes se convierten con frecuencia en los guardianes de sus fronteras[...] En el caso del Derecho, el aislamiento disciplinario ha sido reforzado por el predominio del formalismo jurídico que, al difundir eficazmente la creencia en la separación entre el campo jurídico y los demás campos sociales (v.gr., la política y la economía) ha desalentado o desacreditado la indagación de las conexiones entre lo jurídico y lo social con base en herramientas de análisis tomadas de diversas disciplinas [...] Contra el doble efecto de la ciencia moderna y del formalismo jurídico(que hace parte de aquella) los estudios interdisciplinarios abordan temas y utilizan herramientas de investigación provenientes de múltiples campos de conocimiento, desde la filosofía hasta la sociología, pasando por la historia, la antropología, la ciencia política, la economía y muchos otros. El énfasis de estos estudios radica, entonces, en la relevancia social de los temas de investigación y en el rigor analítico, no en la adecuación de los temas y enfoques a las fronteras disciplinarias" (GARCÍA Y RODRIGUEZ, 2003:17)

Se está entonces de acuerdo con Supiot (2007) en cuanto dice que

"La aspiración a la justicia es un dato antropológico fundamental; puesto que para vivir juntos, los hombres necesitan ponerse de acuerdo en un mismo sentido de la vida. En ese sentido, el Derecho es el texto donde se escriben nuestras creencias básicas: creencia en una significación del ser humano; en el imperio de las leyes o en la fuerza de la palabra dada. Sin ser la expresión de una Verdad revelada por Dios o descubierta por la ciencia, el Derecho es, sin embargo, una herramienta capaz de servir para fines diversos y cambiantes, tanto en la historia de los sistemas políticos como en la historia de las ciencias y de las técnicas. Pero el Derecho es una técnica de la prohibición, que interpone en las relaciones de cada uno con el prójimo y con el mundo un sentido común (una doxa) que lo supera y lo obliga. En efecto, es preciso que cada uno de nosotros esté seguro de un orden existente para poder darles sentido a su propia vida, y a su práctica cotidiana, aunque ésta sea contestataria" (SUPIOT, 2007: 299)

Sin lugar a dudas, es evidente que la ley ha hecho de cada uno de nosotros un sujeto de Derecho.

\footnotetext{
${ }^{21}$ El concepto de Interdisciplinariedad aquí se toma como cualidad de interdisciplinario, es decir, como el proceso de cruzar los límites tradicionales que las caracterizan, y que se produce entre varias disciplinas científicas o culturales o entre varias escuelas de pensamiento por el surgimiento de nuevas necesidades de indagación y/o nuevos enfoques.

${ }^{22}$ A su vez, Transdisciplinariedad aquí se toma como cualidad de transdisciplinar, es decir, el enfoque del conocimiento que trasciende y atraviesa las disciplinas, haciendo énfasis en lo que está entre ellas tanto como en lo que las atraviesa y está más allá de ellas.En ese sentido, la Transdisciplinariedad tiene por finalidad la comprensión del mundo presente desde el imperativo de la unidad del conocimiento. Y se fundamenta en la noción de ir más allá de las disciplinas al aportar una metodología de indagación transdisciplinaria. Significa esto, que la Transdisciplinariedad está constituida por una completa integración teorética y práctica. No rechaza la disciplinariedad,sino que considera el enfoque transdisciplinario complementario al disciplinario; donde cada disciplina aporta elementos para la comprensión de la realidad. En pocas palabras, la Transdisciplinariedad hace emerger de la confrontación de las disciplinas nuevos datos que las articulan entre sí y nos ofrece una nueva visión de la naturaleza y de la realidad.
} 
Sobre este asunto, Supiot (2007) señala que

"Para ser libre, el sujeto primero debe estar ligado ${ }^{23}$ por medio de palabras que lo vinculan con los demás hombres. Los lazos de la palabra y los lazos del Derecho se mezclan para hacer que cada recién nacido acceda a la humanidad, es decir, para que se le atribuya una significación a su vida, en el doble sentido del término, general y jurídico.

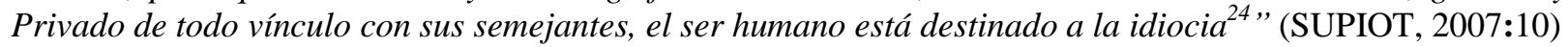

Ahora bien, según acota Supiot (2007) igualmente está amenazado de idiociaquien

"Permanece encerrado en su propia visión del mundo y es incapaz de comprender que hay otras visiones posibles, es decir, quien es incapaz de acordar con sus semejantes una representación del mundo donde cada cual tenga su lugar" (SUPIOT, 2007: 10)

Desde otra mirada, según lo acota Cárdenas (2004) también se pueden plantear los límites y alcances del campo jurídico

"como espacio a través del cual es posible conducir transformaciones estructurales de la sociedad, es decir, de las relaciones económicas dominantes, frente a otras miradas que son escépticas respecto de ese propósito". (CÁRDENAS, 2004)

En ese sentido, Cárdenas (2004) plantea el dilema entre lo que denomina "las posiciones optimistas y pesimistas". Para él

"La primera posición, vería en el Derecho un instrumento capaz de generar transformaciones sociales significativas en la medida en que sus contenidos se puedan desarrollar a cabalidad.En efecto, en el grupo de los optimistas, es posible encontrar pensadores que le otorgan al Derecho una capacidad movilizadora importante frente al orden establecido (v. gr. Boaventura de Sousa Santos). La segunda posición, por el contrario, sitúa al Derecho en la dinámica del devenir social y, en ese sentido es un componente más del orden social, y no 'una variable independiente'. Es una visión escéptica del papel del Derecho, en cuanto las decisiones que en esta materia se toman serían de orden político y responderían, en consecuencia, a intereses específicos, materiales y económicos, de unas determinadas clases sociales o grupos de poder.Desde esta perspectiva, las decisiones que se toman en el orden jurídico, incluso en defensa de los derechos sociales, responden a unos intereses dominantes en lo económico". (CÁRDENAS, 2004)

De manera por demás interesante, Cárdenas (2004) complejiza mucho más esta relación, al indagar en las sutiles interacciones que existen entre estos dos aspectos. Y es en este terreno donde, para él

"Adquiere importancia la discusión con la corriente del Law and Economics, que en buena medida bebe sus fuentes de la llamada Nueva Economía Institucional (NEI). Para esta última, las instituciones -normas, costumbres y acuerdos formales o informales que prevalecen en la sociedad-pueden conllevar importantes costos de transacción para los contratos entre particulares en el intercambio de bienes y servicios, principalmente en ausencia de una clara definición de derechos de propiedad".(CÁRDENAS, 2004)

La discusión del autor se extiende igualmente hacia quiénes desde la otra orilla, ven en el Derecho un instrumento de transformación de las relaciones sociales vigentes. Cárdenas (2004) busca

"Mostrar los límites que el Derecho tiene en sus posibilidades de conducir una transformación significativa de las condiciones que la Economía impone sobre la vida de las personas.Así el Derecho pretenda resolver problemas esenciales de la dignidad humana, la pregunta que está por responderse es de qué sirve tener unas normas que finalmente terminan rebasadas por la realidad económica. Desde esta perspectiva, la evolución de los Derechos Humanos hacia la inclusión de derechos económicos, sociales y culturales se ve sencillamente acotada por las condiciones reales que una sociedad tiene para satisfacer el cumplimiento de tales derechos. Pero al admitir esta condición, hay que señalar que tales limitaciones derivan de la lógica del sistema económico mundial" (CÁRDENAS, 2004)

De otra parte, según Pérez (2004) lo manifiesta

\footnotetext{
${ }^{23}$ Sub-jectum: sujeto sometido

${ }^{24}$ En el sentido etimológico del término griego idios "que está restringido a sí mismo"

118
} 
"A pesar de muchas diferencias de opinión, los ilustrados compartían la creencia de que la conducta individual y la interacción social son ambas susceptibles de estudiarse, entenderse y explicarse con base en la razón.Si el objeto de estudio es el mismo y si la premisa metodológica es válida, las ciencias sociales no pueden escindirse,

aunque se especialicen. Grandes economistas del siglo XIX como John Stuart Mill y Walras tuvieron la misma posición" (PÉREZ, 2004:146)

Y añade Pérez (2004) que

"La disciplina económica fue descrita por Joan Robinson*, quizás con algo de ironía, como una caja de herramientas. Donde lo esencial del contenido de esa caja son dos conceptos: un modelo de racionalidad individual y la noción de que la interacción entre individuos puede entenderse en términos de la operación de un sistema de incentivos. (PÉREZ, 2004:147)

En todo caso Gary Becker [1978, 14] citado por Pérez (2004) reconoció con su habitual prudencia, que

"aún no entendemos buena parte del comportamiento humano, y variables no económicas y las técnicas y hallazgos de otras disciplinas contribuyen de manera significativa a su comprensión. Es decir, si bien el enfoque económico ofrece un marco de aplicación general, muchos de los conceptos y técnicas más importantes provienen y seguirán proviniendo de otras disciplinas" (G. Becker citado por PÉREZ, 2004:155)

Ahora bien, según Pérez (2004) lo trae a colación

"Los grandes avances de la disciplina económica durante el siglo XX se lograron a costa de un distanciamiento cada vez mayor de las demás ciencias sociales. Puede aducirse que éste es consecuencia de la especialización, que a su vez es el precio ineludible del progreso científico. Pero también empobrece la disciplina. Dos grandes economistas llamaron la atención sobre este peligro hace más de medio siglo, cuando la economía se empezaba a autodefinir como algo distinto de otras ciencias sociales. Por un lado, Hayek [1944, 141] afirmó: "Si Usted sabe de economía, y de nada más, será una plaga para la humanidad; no servirá sino para escribir artículos para que los lean otros economistas". Y por otra parte, Jacob Viner (1950:12) advirtió: "Los intereses intelectuales de las personas no son por naturaleza estrechos; se requiere un entrenamiento especial y riguroso para lograrlo. $Y$ quienes han sido entrenados para pensar sólo dentro de los linderos de una disciplina o sólo desde esa perspectiva disciplinaria [...] conocerán muy bien las posibilidades de su disciplina, pero nunca sus limitaciones [...] Samuel Johnson dijo alguna vez [...] "los derechos de reyes y naciones degeneran en asuntos de gramática, si los discuten los gramáticos", [...] ésta es una advertencia a los especialistas para que no reduzcan todos los asuntos a la mera aplicación de su especialidad"(Viner 1950, 1) [Es prudente resaltar que (HAYEK 1944:141 y (VINER 1950:12)son citados por PÉREZ, 2004:155 y s.)

Estamos tan acostumbrados a la compartimentación del conocimiento, que "difícilmente aceptamos que alguien que no es de nuestro dominio ose conocer aquello con lo que tenemos carta de identidad.

Es como si el otro hubiera osado transgredir la frontera disciplinar" (SANJUAN,2006).

Sin embargo, para Estévez (2006) lo que resulta evidente es que

"las transformaciones contemporáneas que se han experimentado, hacen porosas las fronteras de las disciplinas, dando lugar a enfoques más abiertos”. (ESTÉVEZ,2006).

Siendo indiscutible que "cada época desarrolla la ciencia que necesita y la ciencia que puede" (Maldonado, 2016), resulta interesante constatar que asistimos a una época de una impresionante complejidad de la sociedad, el mundo y la naturaleza.

Nuevas ciencias, nuevos enfoques, nuevas metodologías, nuevos lenguajes y nuevas relaciones disciplinares emergen y se dan a la tarea, al mismo tiempo, de comprender los hechos, las circunstancias y las acciones.

Sin embargo, esto ocurre en entornos cada vez más turbulentos e inestables, en los que detrás del fantasma de los intereses por los cuales los agentes sociales compiten en la sociedad, se evidencianlos procesos y centros de poder y decisiónque los constituyen. Así, en efecto es posible percibir que el espacio social y/o la organización donde uno se sitúa resultan afectados, positiva o negativamente, por procesos y/o centros de poder y de decisión más o menos ajenos a nuestro control.Ahora bien, de hecho, lo que preocupa no es que los factores constitutivos del contexto capaces de afectar a una sociedad, a sus instituciones y a sus organizaciones sean ciertamente ajenas a ellas, sino, y fundamentalmente, el hecho de que dichas fuerzas estén parcial o totalmente por fuera de su control.

Como se puede ver, en el campo que nos ocupa, del binomio derecho-economía, paulatinamente se van abriendo sus fronterasde conocimiento hacia lo que se ha dado en llamar el complejo trinomio derecho-economía-sociedad 
(DES).Al respecto, Silva García (2000) lo explica de manera sencilla en apretada síntesis que he decidido transcribir completa por su lucidez.

El mencionado autor se expresa de la manera siguiente:

"Para alguien que se ocupa de la Sociología Jurídica, como ocurre en mi caso, el Análisis Económico del Derecho es un ámbito que no resulta del todo extraño o ajeno. En pocas palabras, de acuerdo con una visión general y comúnmente aceptada, la Sociología Jurídica trata de las relaciones entre Derecho y Sociedad, escenario dentro del cual cabe considerar lo económico como uno de los aspectos que, en el lugar de la Sociedad, hacen parte de esa relación. Lo económico es, entonces, parte del objeto de estudio de la Sociología del Derecho, obviamente tema central de la Ciencia Económica y, también, materia de estudio de varias disciplinas más, aunque en todos los casos difieren las formas e intensidad de su conocimiento. De otra parte, las teorías económicas han influido en la construcción de las doctrinas de todas las ciencias sociales. En consecuencia, para el caso específico de la Sociología del Derecho, la importancia de la economía es doble si se reconoce a la disciplina en tanto fuente teórica y a su problemática como parte de su objeto de estudio [...] Desde luego, la Economía no es la única faceta contemplada en el análisis sociojurídico, aunque una de las corrientes de la Sociología del Derecho haya adoptado esa esfera como componente fundamental para enfrentar el trabajo teórico y la investigación, según VincenzoFerrari lo manifiesta en su libro Funciones del Derecho. Se ha señalado que una de las variables principales que inciden en el contexto que modela los conflictos sociales objeto de tratamiento por el Derecho es la organización social, la cual es examinada según dos perspectivas generales sobre el sistema social. En una de ellas se considera que la organización social es influida por una multiplicidad de factores con distinta relevancia cualitativa.

En la otra parte se plantea que existe un factor cuantitativo predominante en la configuración de la organización social, que muchas veces es definido como el económico o, en forma más específica, como el modo de producción [...] En todo caso, la estructura económica es uno de los pilares fundamentales que contribuyen a perfilar cualquier organización social y, por ende, lo económico puede ser introducido de muchas maneras en la relación DerechoSociedad.Por ejemplo, examinando el impacto o los costos económicos de las medidas legales, las tentativas del Derecho para inducir transformaciones en las relaciones económicas, la ineficiencia de las normas jurídicas, incluidas las políticas públicas, neutralizadas por las condiciones económicas y centros de poder económico, la ocurrencia de cambios jurídicos provocados por variables económicas, etc. Esos temas son asuntos que le incumben a la Sociología del Derecho, pero igualmente han sido tratados por los economistas. El Análisis Económico del Derecho y de las instituciones que le son propias, como la justicia, involucra a diferentes disciplinas dotadas con variadas herramientas teóricas, concepciones y, sobre todo, distintos énfasis para el abordaje de su estudio. Por tanto, el Análisis Económico del Derecho, que yo definiría como una línea de teorización y pesquisa interdisciplinaria, no es patrimonio exclusivo de la Economía, aunque a esa ciencia le corresponda desempeñar al respecto un papel crucial" (SILVA GARCÍA G, 2000).

Ahora, como lo manifiesta Durán (1992) si bien

"Todo este planteamiento lleva a Becker a conformar la necesidad de una aproximación cuantitativa a variables que no son propiamente pecuniarias; y de ese modo, poder constatar el estudio, desde la perspectiva económica, de cuestiones que tradicionalmente no han sido consideradas como tales" (DURÁN; 1992: 93).

Sin embargo, de acuerdo con la profesora española Durán (1992), en su opinión

"Para hacer operativa esa propuesta, por ejemplo, desde la perspectiva de las políticas públicas, habría que distinguir entre lo que significa un estudio de las consecuencias sociales que tiene una acción típicamente económica; del intento de interpretar económicamente acciones humanas, que al ejecutarse pueden tener de hecho repercusiones económicas, pero en ningún caso identificables con una idea a priori económica”(DURÁN; 1992: 93).

En el ámbito de la materialización de las transformaciones y aperturas del Derecho, su sentido regulativo ha devenido asociado con el desarrollo de instrumentos de política para la intervención económica.

Calvo García (1998) señala que siendo más rigurosos

"quizá habría de señalarse que el Derecho regulativo conecta con la tradición jurídico-burocrática de control de ciertos ámbitos de la actividad económica y los mercados desde comisiones o agencias independientes en E.E.U.U” (CALVO GARCÍA, 1998:99)

Es obvio, entonces, como García Amado (1998) bien lo acota 
"que según la noción del Derecho que se maneje, variará la importancia que se otorgue a la Sociología Jurídica como disciplina que pueda aportar conocimientos válidos para una más precisa aprehensión de la realidad jurídica" (GARCÍA AMADO, 1998: 181)

Sí por lo general, como García Amado (1998) bien señala

"la Sociología Jurídica es, en su más simple y elemental caracterización, el campo de análisis del Derecho como hecho, es decir, de la realidad fáctica, pragmática, vivencial y social que el Derecho representa [...] cabe pensar que entre el estatuto de la Sociología Jurídica y la relevancia que se le otorgue como disciplina útil para el jurista, por un lado, y la definición de lo jurídico que manejemos, por otro, habrá una conexión fundamental" (GARCÍA AMADO, 1998: 181)

De este modo, nos encontramos frente a una apertura disciplinar importante para el estudio y análisis de las estructuras normativas, sociales, jurídico-económicas y de poder.

Una iluminadora aplicación investigativa del enfoque de Derecho, Economía y Sociedad (DES) lo constituye la contaminación hídrica, circunstancia recurrente hoy en nuestro país, países vecinos y el mundo.

De tal modo, en la medida en que un agente social produce emisiones que disminuyen la calidad del agua, actividad con la cual termina por afectar a la comunidad que utiliza el mismo recurso, se genera con dicha acción una externalidad negativa. Este tipo de impacto ocurre, toda vez que dicho agente utiliza el recurso hídrico como vertedero de sus desperdicios, sin asumir los costos que ello representa.

Se desprende, entonces, de tal acto, que mientras dicho agente lo siga haciendo, tales costos los asume la sociedad, bien sea de manera directa, destinando recursos económicos a descontaminar; o de manera indirecta, incrementando sus costos de salud para contrarrestar enfermedades originadas por dicha contaminación o en caso extremo, teniendo que desplazarse hacia lugares no contaminados.

Cuando la emisión permanece, la sociedad o laspersonas que la integran deben disponer recursos económicos para mitigar el cambio en la calidad ambiental o el daño causado por las emisiones.

Estos efectos externos dan como resultado que los costos sociales son mayores que los costos asumidos por el agente que originó la contaminación.

Por otra parte, las migraciones se han convertido también en objeto de investigación desde este enfoque, en su doble faz emigración/inmigración sobre las ilusiones del emigrante, los padecimientos del inmigrante, la regulación, las condiciones de vida, y la situación laboral en las sociedades receptoras; y el impacto de las remesas y su regulación, en las sociedades emisoras.

\section{Conclusiones}

Se asume que el Derecho "no es una disciplina autónoma, autosuficiente sino una disciplina con un objeto específico" ${ }^{25}$, que no puede disolverse solo en Economía o en cualquier otra disciplina, sino que tal como Botero (1997)lo señala"encuentra su base de conocimiento en la ciencia social, a la cual a su vez el Derecho tributa un importante aporte" (Ibídem).

Significa eso que "no puede estudiarse Sociología o cualquier otra disciplina social ignorando el Derecho" (Ibídem). Se llega, por tanto, a la conclusión que "la interdisciplinariedad es un imperativo para el estudio y la práctica de las disciplinas sociales" (Ibídem).

El desplazamiento de las fronteras del Derechoy la Economía hacia lo social, la relevancia de la incorporación de lo jurídico y de lo económico en la sociedad como aspectos definitorios, variará según la visión que se tenga de las mencionadas disciplinas. Así, como García Amado (1998) acota "la presencia de lo social será mayor en las descomposiciones existenciales que en las analíticas" (GARCÍA AMADO, 1998:185).

Es posible, entonces, que las Facultades de Derecho hayan estado por décadas ajenas a los signos y cambios de los tiempos, y que por tanto, raramente sean sus impulsoras. Quizás eso se deba al hecho de que los cambios se originan y ganan vigencia en la sociedad, y los Programas de Derecho han estado de espaldas a esas manifestaciones transformadoras.

Quiere eso decir, que la investigación de los hechos e incidencias sociales del Derecho y la Economía, será más relevante para la caracterización de los elementos definitorios de lo jurídico y lo económico, cuanto más se ubiquen,

\footnotetext{
${ }^{25}$ BOTERO, D. (1997) Teoría social del Derecho UNIVERSIDAD NACIONAL DE COLOMBIA, FACULTAD DE DERECHO, CIENCIAS POLÍTICAS Y SOCIALES.
} 
estos dos campos, en el mundo de los datos fácticos. Esta concreción del análisis del orden jurídico-económico contemporáneo, desde la perspectiva de las ciencias sociales, también es análisis sociojurídico.

No en vano, ha dado pie a la paulatina expansión de una importante vertiente de la Sociología Jurídica:Derecho, Economía y Sociedad (DES) como amplio campo de investigación que ha producido, tanto en América Latina como en Estados Unidos y Europa (particularmente en Portugal, España, Alemania e Italia), algunas de las contribuciones interdisciplinarias y/o transdisciplinarias más interesantes al estudio del Derecho, toda vez que combinan los aportes no sólo de la Sociología, sino también de la Ciencia Política, la Antropología, la Historia, la Teoría Social y la Economía.

Es precisamente el carácter abierto al mundo, el que da una inmensa plasticidad al comportamiento humano, permitiendo a las personas adecuar su juego a las circunstancias cambiantes del entorno.

Por eso cuando en una situación entran elementos nuevos, o cuando ésta es completamente novedosa, las personas encuentran estrategias de acción ingeniosas para resolver con gran "sentido del juego" los problemas que se les plantean.

Como Rodríguez (2002) lo señala "la convergencia de diversos asuntos y metodologías en el estudio de las Instituciones Jurídicas, Económicas y Políticas tomadas en el contexto en el cual operan, es el rasgo diferenciador" (RODRÍGUEZ, 2002).

Esto, toda vez que facilita "la comparación entre el Derecho tal cual se expresa en los Códigos, Leyes y directrices de Política Pública y el Derecho tal cual opera en lasvivencia y prácticas cotidianas en la sociedad mediadas por la Economía, como objeto de indagación" (Ibídem).

Esta variedad de posibilidades,"permite no sólo tener una perspectiva crítica de las experiencias, prácticasjurídicoeconómico-sociales y de los impactos de las diversas instituciones, sino tener en cuenta la diversidad de contextos sociales en los que operan, para no pasar de largo aspectos esenciales en el diseño de políticas públicas”(Ibídem).

Resulta interesanteconstatar que asistimos a una época de una impresionante complejidad de la sociedad, el mundo y la naturaleza.

En ese complejo escenario, emergen nuevas ciencias, nuevos enfoques, nuevas metodologías, nuevos lenguajes y nuevas relaciones disciplinares, las cuales se dan a la tarea, al mismo tiempo, de comprender los hechos, las circunstancias y las acciones, en entornos cada vez más turbulentos e inestables.Y en esos entornos, detrás de los procesos y centros de poder y decisión que los constituyen aparece el fantasma de los intereses por los cuales los agentes sociales compiten en la sociedad.

En consecuencia, una de las tareas de los estudios e investigaciones de lo que se ha dado en llamar Derecho, Economía y Sociedad (DES), en palabras sencillas es precisamente la de abordar los ordenamientos jurídico-político-económicos actuales en esa clave, e identificar las variables, los procesos y los centros de poder y decisión que los influencian y que a no dudarlo, son en el fondo y en última instancia el gran secreto de los conflictos contemporáneos.

\section{Lista de Referencias}

AARNIO, A.; GARZÓN VALDÉS, E.; UUSITALO, J. (comps) (1997) La normatividad del Derecho, Gedisa, Barcelona.

AÑóN, M. J. (2001) Igualdad, diferencias y desigualdades, Fontamara, Ciudad de México. et alia (1998) Derecho y Sociedad, Tirant lo Blanch Libros, Valencia (España)

BECKER, G. (1976) The Economic Approach to Human Behavior, Chicago, University of Chicago Press.

BOTERO, D. (1997) Teoría social del Derecho UNIVERSIDAD NACIONAL DE COLOMBIA, FACULTAD DE DERECHO, CIENCIAS POLÍTICAS Y SOCIALES.

BOURDIEU, P. (1991) "Les juristes, gardiens de l'hypocrisie collective", en F. Chazel et J. CommailleNormes juridiques et regulation sociale, París, LGDJ.

BOURDIEU Y WACQUANT (1995) Respuestas. Por una antropología reflexiva, Méjico, Grijalbo.

BOURDIEU, P. (2000) "Elementos para una sociología del campo jurídico" en La fuerza del Derecho, Bogotá, Ediciones Uniandes/Instituto Pensar/ Siglo del Hombre Editores.

BRIGIDO, A. M. (2002) "Bourdieu y sus aportes a una sociología del campo jurídico", Argentina, Revista Páginas N 2-3(2) Septiembre de 2002, U. N. C, pp 119-122.

CALVO GARCÍA, M. (1998) "Paradojas regulativas: las contradicciones del Derecho en el Estado Intervencionista" en Añon M. J., Bergalli R, Calvo M, Casanovas P. Derecho y Sociedad, Valencia (España) Editorial Tirant lo Blanch. 
CALVO GARCíA, M. (2005) Transformaciones del Estado y del Derecho, Bogotá, Universidad Externado de Colombia, Colección Sociología Jurídica y Política $\mathrm{N}^{\circ} 1$.

CARDENAS, M. E. (2004) Justicia pensional y neoliberalismo. Un estudio de caso sobre la relación derecho y economía. Bogotá, ILSA, EdicionesÁntropos, Textos de aquí y de ahora.

DORFMAN, J. y otros, eds. (1963) Institutional Economics, Veblen, Commons and Mitchell reconsidered, University of California Press; Berkeley.

DURÁN, P. (1992) Una aproximación al Análisis Económico del Derecho, Granada, España, Editorial Comares.

DURKHEIM, E (2007) De la división du travail social, reimpresión PUF, París.

ESTÉVEZ, J. A. (2006) El revés del Derecho, Bogotá, Universidad Externado de Colombia, Colección Sociología Jurídica y Política 3

FERRARI, V. (2006) Derecho y Sociedad. Elementos de Sociología del Derecho, Bogotá, Universidad Externado de Colombia.

(2014) Funciones del Derecho, Bogotá, Universidad Externado de Colombia.

FLACHSLAND, C. (2005) Pierre Bourdieu y el capital simbólico, Buenos Aires, Editorial Campo de Ideas SL.

FOUCAULT, M. (1972) The archaeology of knowledge and the discourse on language, New York, Pantheon.

GARCÍA AMADO, J. A. (1998) "El elemento social en las definiciones del Derecho", en Añon, M. J., Bergalli R, Calvo M, Casanovas P. Derecho y Sociedad, Valencia (España) Editorial Tirant lo Blanch.

GARCÍA MÁYNEZ, E. (1993) Positivismo jurídico, realismo sociológico y iusnaturalismo, Fontamara, Ciudad de México.

GARCÍA VILLEGAS, M. y RODRÍGUEZ, C.A. (2003) "Derecho y sociedad en América Latina: propuesta para la consolidación de los estudios jurídicos críticos" en MAURICIO GARCÍA VILLEGAS Y CÉSAR A. RODRÍGUEZ (EDS) DERECHO Y SOCIEDAD EN AMÉRICA LATINA: un debate sobre los estudios jurídicos críticos, Bogotá, Instituto Latinoamericano de Servicios legales Alternativos/ILSA y UNIVERSIDAD NACIONAL DE COLOMBIA, Facultad de Derecho, Ciencias Políticas y Sociales.

GIRALDO ÁNGEL, J. et alia (2002) Metodología y técnica de la investigación sociojurídica, Ediciones Librería del Profesional, Bogotá.

HABERMAS, J. Teoría de la acción comunicativa, traducción al castellano M. Jiménez, Madrid, Taurus, 2 volúmenes, 1987-1988.

"Legitimidad a través de la legalidad", traducción al castellano M. Jiménez, en Doxa, vol V, pp. 21 a 47.

IBÁÑEZ, J. W. (2011) Análisis Económico del Derecho. Método, investigación y práctica jurídica. Barcelona, Bosh Editor.

JIMÉNEZ, I. (1997) Pierre Bourdieu Capital cultural, escuela y espacio social, México, Siglo XXI Editores, Colección Sociología y Política.

JUDT, T (2010) Algo va mal, Taurus, Madrid

LISTA, C. y BRIGIDO A. M. (2002) "La enseñanza del derecho y la formación de la conciencia jurídica", Córdoba, Argentina, Sima Editora, octubre de 2002.

MALDONADO, C.E. (2016) Complejidad de las Ciencias Sociales. Y de otras Ciencias y Disciplinas, Bogotá, Ediciones Desde Abajo.

MONTORO BALLESTEROS, M. A. (1999) "Incidencia del análisis económico del derecho en la teoría jurídica: la reducción del derecho a regla técnica", Universidad de Navarra, Revista Persona y derecho: de fundamentación de las Instituciones Jurídicas y de Derechos Humanos, ISSN 0211-4526, Nº 40.

PARAMIO, L. (2000) "Decisión racional y acción colectiva" Leviatán79, pp. 65-83

(2002) "La globalización y el malestar en la democracia" Revista Internacional de Filosofía Política 20, pp 5-23

PEREZ, (2004) "La economía en el panorama de las ciencias sociales. Variaciones sobre un tema de Bejarano" Bogotá, Revista Cuadernos de Economía 40, 2004

SANJUAN, M. (2006) Rompiendo fronteras disciplinares, en www.madridmasd.org/blog/complejidad/2006/01/28/12897 consultado en Junio 27/2018 a las 22:15

SILVA GARCÍA G. (2000)“Una revisión del análisis económico del derecho. Una lectura crítica a propósito de la obra Crimen e impunidad”, Bogotá, Revista Economía Institucional N² 2, Primer semestre/2000

SUPIOT, A. (2007) Homo juridicus. Ensayo sobre la función antropológica del derecho, Buenos Aires, Editorial Siglo XXI, Colección Sociología y Política.

TÉlleZ IREGUI, G. (2002) Pierre Bourdieu Conceptos básicos y construcción socioeducativa. Claves para su lectura, Bogotá, Universidad Pedagógica Nacional.

WEBER, M. (1979) Economía y sociedad. Esbozo de sociología comprensiva, J. WINNCKELMANN (ed.), J. MEDINA et ál. (trads.) México, Fondo de Cultura Económica. 